\title{
PRÁCTICAS ECONÓMICAS DE MIGRANTES CHINOS Y SENEGALESES EN LA CIUDAD DE CÓRDOBA
}

\author{
Economic practices of Chinese and Senegal migrants \\ in the city of Córdoba
}

Eduardo Rodríguez Rocha ${ }^{1}$

\begin{abstract}
Resumen. El presente artículo constituye un análisis exploratorio de dos prácticas económicas personificadas por migrantes de origen chino y senegalés que se han vinculado a la economía de los bazares en el contexto de la ciudad de Córdoba, Argentina. Concretamente, el trabajo describe cómo se articulan, por un lado, las iniciativas económicas de los comerciantes chinos que regentean bazares/tiendas en los que venden principalmente productos procedentes de China, con otras personificadas por vendedores ambulantes senegaleses que revenden en las calles de la ciudad, algunas de las mercancías comercializadas en los bazares regenteados por los comerciantes chinos. El argumento principal del artículo es que estos nexos comerciales producen experiencias económicas complementarias, aunque profundamente desiguales entre sí. En el artículo se instrumenta una perspectiva al mismo tiempo global y des-etnizada, que permite abordar fenómenos relacionados a los procesos a través de los cuales diversos grupos de migrantes transnacionales se vinculan, "desde abajo", a la economía global no hegemónica. El artículo hace uso de datos provistos por un trabajo de campo de corte etnográfico, que desde el mes de enero del 2016 hasta la fecha, se ha llevado a cabo en el centro histórico de la Ciudad de Córdoba.
\end{abstract}

Palabras clave: migración transnacional, China, Senegal.

Abstract. This article is an exploratory analysis of two economic practices personified by Chinese and Senegalese immigrants, who have been linked to the bazaar economy in the context of the city of Cordoba, Argentina. Specifically, the paper describes how the economic initiatives of Chinese traders who run bazaars / shops in which they mainly sell products from China are articulated, on one hand, with the initiatives embodied by Senegalese street vendors whom resell some of the goods traded in the bazaars ruled by the Chinese merchants. The main argument of the article is that these links produce complementary economic experiences, although deeply unequal to each other. In the article, a global and un-ethnicized

1 CONICET/Centro de Investigaciones y Estudios sobre Cultura y Sociedad. Córdoba, Argentina. 
perspective is implemented, which allows us to deal with social phenomena related to the processes through which diverse groups of transnational migrants are linked "from below" to the non-hegemonic global economy. The article uses data provided by an ethnographic fieldwork carried out in the historic center of the City of Cordoba, since January 2016.

Keywords: transnational migration, China, Senegal.

\section{Introducción}

El estudio de los movimientos migratorios procedentes de países extraregionales en Argentina constituye una línea de investigación incipiente en este país. De tal forma, son pocos los trabajos que hayan abordado dichos procesos que, en términos proporcionales, son minoritarios en la composición de las corrientes migratorias centrales. En este articulo pretendo aportar a dicho campo de estudios, a través de la caracterización de dos prácticas económicas personificadas por personas inmigrantes originarias de países de diversas subregiones de Asia y África vinculadas al entramado comercial de los bazares polirubro, en el contexto del centro histórico de la ciudad de Córdoba, Argentina.

Específicamente, en esta oportunidad me centro en las actividades llevadas a cabo por comerciantes de origen chino, que al mismo tiempo son inversores y trabajadores de sus propios negocios, en los que se venden, principalmente, juguetes, artículos electrónicos, textiles, de perfumería, bijouterie y una infinidad de productos baratos de plástico. Como explico en este documento, estos bazares polirubro se incrustan a sólidos encadenamientos transnacionales, condición que ha contribuido en su consolidación económica. Adicionalmente, abordo las relaciones que establecen estos comerciantes mayoristas con otros vendedores ambulantes de origen senegalés. Se ha podido apreciar que buena parte de los comerciantes senegaleses son revendedores minoristas de las mercancías adquiridas en los bazares regenteados por los migrantes chinos. En tanto, son asumidos como comerciantes que desarrollan estrategias de supervivencia reproducidas a costa de los bazares mayoristas.

El objetivo principal de este trabajo es realizar, haciendo uso de información etnográfica recolectada durante los meses de enero y noviembre del 2016 en la zona céntrica de la ciudad de Córdoba, una descripción que sea capaz de ilustrar cómo se configuran y relacionan ambas condiciones de producción. El supuesto que subyace a este objetivo es que estas relaciones comerciales producen experiencias económicas desiguales, aunque complementarias. Para cumplir con el objetivo, este trabajo se adscribe a una perspectiva teórica que sostiene que aquellas iniciativas económicas personificadas por migrantes transnacionales, específicamente las vinculadas a la economía global no hegemónica, deben estudiarse a partir de las experiencias llevadas a cabo desde 
abajo por los actores "micro" de la globalización. Las perspectivas "desde abajo" se han desarrollado recientemente por diversos trabajos de corte sociológico y antropológico que han demostrado cómo los sectores subalternos generan iniciativas económicas alternativas a las formaciones económicas hegemónicas globales ${ }^{2}$. Algunas de estas propuestas enfatizan los aspectos políticos y culturales de dichos procesos ${ }^{3}$. En cambio, la globalización desde abajo a la que se adscribe este trabajo se enfoca en los mecanismos estrictamente económicos que despliegan diversos agentes para sobrevivir ${ }^{4}$. Se trata de un enfoque que permite acercarse a las lógicas compuestas por trabajadores en movilidad que poseen capitales económicos pequeños que activan transacciones informales, en ocasiones ilícitas o cuasi-legales. Es decir, se sostiene que la producción de la economía desde abajo es personificada, mayoritariamente, por

comerciantes del segmento pobre de la globalización, que compran mercancías usadas o las copian fuera del control legal y transportan en contenedores o en sus maletas a través de los continentes y de las fronteras para que los vendedores callejeros los vendan a precios mínimos sin que nadie pregunte por su procedencia ${ }^{5}$.

Siguiendo esta línea analítica, en este trabajo acuño la perspectiva de las cadenas de valor que como se ha argumentado en otros lugares, resulta pertinente para describir la gama de actividades requeridas para que un producto o servicio se conciba desde su confección, pasando por los procesos de distribución y entrega a los consumidores finales ${ }^{6}$. Mediante esta perspectiva me limito a explorar la caracterización de dos eslabones cruciales de una cadena mundial de mercancías que tiene su origen en las fábricas del sureste de China y su fin en las prácticas de consumo en las calles de la ciudad de Córdoba. Me refiero a los bazares mayoristas polirubro y a las actividades relacionadas con la venta ambulante de dichas mercancías. Como he señalado más arriba, para esta descripción hago uso de material etnográfico compuesto por un trabajo de campo de corte etnográfico realizado en la zona comercial del centro de Córdoba en el que pude registrar mediante observación no participativa los modos de articulación entre ambas actividades económicas. Estas observaciones se han realizado en los lugares de trabajo, en diversos puntos del centro de la ciudad. Asimismo, realicé decenas de entrevistas a estos comerciantes, lo que me permitió obtener información de primera mano.

2 RIBEIRO, Gustavo. Non hegemonic globalizations. Alternativa transnational Processes and agents; ALBA VEGA et alii. La globalización desde abajo. La otra economía mundial.

3 GAGO, Verónica. La Razón Neoliberal; APPADURAI, Arjun. Grassroots Globalization and the Research Imagination.

4 ALBA VEGA et alii, op. cit.

5 Ibidem, p. 27.

6 AGUIAR, José Carlos. "Vienen de China". Los CD piratas en México desde una perspectiva transnacional. 
El trabajo de campo se dividió en dos momentos. En primer lugar, de observaciones y recorridos diarios por este espacio urbano. En segundo lugar, se dio paso a los testimonios brindados por los sujetos que decidieron ser parte de este trabajo. Su obtención se generó a partir de la técnica de la bola de nieve. Durante el trabajo de campo se desarrolló una mirada des-etnizada, a partir de la cual las relaciones comerciales establecidas por diversos comerciantes que participan de la globalización desde abajo se constituyeron en las unidades de análisis. De tal forma, las características étnico-nacionales de los sujetos no fueron tomadas en cuenta para el recorte del objeto de estudio ${ }^{7}$. Es decir, lo "étnico" es concebido menos como un criterio de distinción analítica que como una construcción social que, según la estructuras de poder predominantes, es experimentada de manera diversa por los agentes sociales involucrados. De tal manera, el criterio inicial para identificar quienes serían parte del trabajo fue establecer contacto con comerciantes nacidos en el extranjero que se hubiesen asentado en la ciudad durante los últimos treinta años. El contacto se concretó personalmente en los lugares de trabajo de los informantes. Dado que los primeros contactos se establecieron con bazaristas chinos, éstos derivaron a sus respectivas redes y referentes comunitarios. De manera similar, se obtuvieron testimonios de comerciantes senegaleses, indios y paquistaníes dedicados a la economía del bazar y a la economía informal callejera. Puesto que todos los informantes senegaleses eran practicantes religiosos, una buena cantidad de entrevistas fueron realizadas en sus centros culturales islámicos, ubicados también en esta zona de la ciudad. Tal oportunidad significó la posibilidad de entrevistar a migrantes musulmanes marroquíes, sudafricanos y argelinos, todos ellos dedicados a la economía del comercio en baja escala. Hasta el momento se han realizado 26 entrevistas individuales. Asimismo, decenas de charlas informales terminan de conformar un trabajo de campo etnográfico aún abierto.

El diseño de las entrevistas ha procurado captar, en primer lugar, información relativa a la posición de origen social, a la trayectoria educativa y al itinerario ocupacional de los entrevistados. En segundo, a sus percepciones relativas a las experiencias de resolución de vida actuales. En tercero, a las predisposiciones, expectativas y aspiraciones de corto y mediano plazo. Si bien el grueso de los testimonios ha sido provisto por jóvenes varones, también algunos adultos fueron entrevistados. Todos ellos veteranos con largas trayectorias ocupacionales desarrolladas en decenas de ciudades a lo largo y ancho del mundo.

Como he señalado, en este artículo abordo el estudio de las relaciones comerciales entrelazadas entre migrantes de origen chino y senegalés. Por

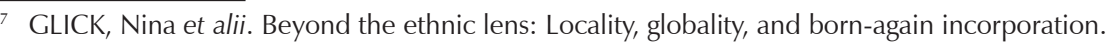


razones de espacio, no abordo las formas de participación económica de otros migrantes extra-regionales que han sido entrevistados en la investigación. De la misma manera, me limito a la descripción de las condiciones objetivas que configuran a las iniciativas económicas personificadas por los comerciantes chinos y senegaleses. Puesto que este trabajo se enmarca en una investigación en curso y de mayor alcance analítico, en ulteriores oportunidades deberán atenderse los aspectos relacionados a los procesos de construcción subjetiva de quienes experimentan estas condiciones de producción.

El trabajo se estructura de la siguiente manera. En el próximo apartado presento los ejes teóricos a partir de los cuales me he servido para captar las prácticas económicas bajo estudio. Así, en primer lugar explico las razones analíticas que justifican la adscripción a las miradas "desde abajo", en demérito de otras miradas que abordan las actividades económicas de los migrantes en las sociedades de recepción. De tal forma, me centro en la perspectiva de las cadenas mundiales de mercancías. En el tercer apartado abordo brevemente y de forma no exhaustiva el papel de China como actor clave en la estructuración de los encadenamientos comerciales mundiales, centrándome especialmente en los relacionados a las mercancías de bazar. En el cuarto abordo las relaciones complementarias y desiguales que han establecido en el centro histórico de la Ciudad de Córdoba, las iniciativas económicas personificadas por los comerciantes chinos que manejan bazares mayoristas y los vendedores ambulantes senegaleses. Como he explicado, se trata de una descripción "objetivista", por lo que no avanzo en las representaciones sociales construidas por los actores involucrados en dichos procesos. Finalmente, en el último apartado presento los aportes principales del trabajo, sus limitaciones, y los ulteriores aspectos y líneas de investigación a ser desarrollados.

\section{La perspectiva centrada en las cadenas mundiales de mercancías}

Existe un extenso material bibliográfico sobre las actividades económicas de los migrantes extranjeros en los países receptores. Es posible distinguir dos grandes líneas: aquella que analiza las actividades micro-empresariales de los migrantes y la que se centra en las estrategias de supervivencia desarrolladas en el sector informal urbano. Con respecto a la primer línea, se erigen tres perspectivas: el enfoque cultural, el del enclave étnico y el de la incrustación mixta $^{8}$. Con respecto a la segunda, se han ensayado desde finales de la década de 1960, cuatro interpretaciones: los trabajos adscriptos a la teoría de la modernización desarrollista, a la teoría de la dependencia, el enfoque del

\footnotetext{
${ }_{8}$ Algunos detallados análisis que repasan estos enfoques teóricos son: SOLÉ, Carlota, PARELLA, Sonia, CALVACANTI, Leonardo. El empresariado inmigrante en España; y CHIN, Ku-Sup, YOON, In-Jin, SMITH David. Immigrant Small Business and International Economic Linkage.
} 
sector informal urbano de la Organización Internacional del Trabajo y, más recientemente, una lectura neo-marxista de la marginalidad económica9 ${ }^{9}$.

Sin desestimar las diferencias teórico-metodológicas de estas posturas, e inclusive ideológicas, es posible enunciar que éstas conciben a las actividades económicas de los migrantes como prácticas realizadas dentro de los segmentos laborales inscriptos en los estados-nacionales. En contraste, en este trabajo se argumenta que es deseable que los análisis que busquen explorar las prácticas económicas contemporáneas de los migrantes internacionales, deben asumir que los intercambios de bienes materiales y de dinero entre los agentes involucrados en los procesos de resolución de vida alternativos a la economía hegemónica no se enmarcan en los denominados sectores "formales" e "informales" de las economías nacionales. En todo caso, como se ha destacado en otros trabajos, las prácticas vinculadas a la globalización desde abajo frecuentemente se desarrollan en sistemas y por culturas translocales a partir de las cuales los sujetos pueden hacer caso omiso del poder regulatorio y normativo de los Estados-nación, o evitarlo, y crear unidades político-culturales complejas y relativamente independientes ${ }^{10}$.

Una manera de describir y analizar dichos mecanismos de resolución de vida es mediante el estudio de las cadenas mundiales de mercancías. El concepto alude a toda una gama de actividades que tanto las empresas y los trabajadores llevan a cabo para que las mercancías se produzcan, circulen y lleguen a un destino final11. Es decir, el foco está puesto en la movilización internacional de mercancías a través de distintas actividades comprometidas en el proceso. Actividades que a su vez se pueden concebir como eslabones que configuran a los encadenamientos, que en muchas ocasiones se reproducen en las borrosas fronteras de lo legal, lo ilegal y lo ilícito ${ }^{12}$. Así, se ha destacado el papel de productores, inversores, abogados, agentes aduanales, funcionarios de gobierno, transportistas, agentes comerciales clandestinos, vendedores y puntos de venta formal e informal vinculados a la globalización desde abajo ${ }^{13}$.

Precisamente, las cadenas mundiales de mercancías de bazar absorben en buena medida las lógicas relacionadas a la globalización desde abajo. En primer lugar, porque este entramado está organizado globalmente a partir de su imbricación a la economía no hegemónica. En segundo lugar, porque desde hace aproximadamente 20 años la participación de los migrantes extranjeros

\footnotetext{
9 Keith Harth, en el Prefacio del libro compilado por ALBA VEGA et alii, op. cit., ofrece una apretada síntesis de estas tradiciones de investigación.

${ }^{10}$ RIBEIRO, Gustavo. Economic Globalization from Below; MEZZADRA, Sandro. Capitalismo, migraciones y luchas sociales. La mirada de la autonomía.

11 AGUIAR, op. cit.

12 Ibidem.

13 Ibidem.
} 
en la economía del bazar se ha multiplicado significativamente. Esto ha sido documentado en distintas ciudades europeas, norteamericanas y asiáticas ${ }^{14}$. Para comprender ambos aspectos, es preciso señalar el papel de la República Popular China (en adelante China) como actor clave en la producción de lógicas económicas no hegemónicas.

\section{El papel de China en la economía del bazar}

A partir de 1978 15 , la internacionalización económica de China ha tenido efectos profundos en algunos países del sur-global. El país asiático no sólo se ha consolidado como la segunda economía global y como el primer exportador mundial de manufacturas, sino que ha generado intereses comerciales de diversos actores no hegemónicos. Especialmente en la zona costera en donde se ubican las provincias de Fujian y Guangdong, se ha instalado un millar de fábricas que producen una variedad inmensa de mercancías ilegales no certificadas, así como fábricas legales que se relacionan con redes que contrabandean legal o ilegalmente dicha producción a lo largo y ancho del mundo ${ }^{16}$. Esta ambigua capacidad productiva ha dado cauce a la proliferación de diversas cadenas de mercancías que circulan por distintas rutas hasta llegar a los destinos de consumo, especialmente en localidades y países en los que la economía de la globalización desde abajo es una fuente de recursos social y culturalmente legitimada ${ }^{17}$.

El surgimiento y la intensificación de las rutas por las que circulan las cadenas mundiales de mercancías constituyen una condición estrechamente vinculada a la promoción hacia el exterior de la cultura socialista impulsada por el gobierno chino a través de la construcción de puentes económicos con las diversas organizaciones comerciales de ultramar $^{18}$. Ello ha contribuido en la formación de una diáspora global de migrantes que se han incorporado a los países de llegada a través de su participación en los múltiples componentes de

${ }_{14}$ TSENG, Yen. Beyond "Little Taipei": The Development of Taiwanese Immigrant Businesses in Los Angeles; YOON, In-Yin. The Changing Significance of Ethnic and Class Resources in Immigrant Businesses; LÓPEZ SALA, Ana, SÁNCHEZ, Valeriano. Los comerciantes sindhis en Canarias; VUDDAMALAY, Vasoodeven. Empresarios indios en Francia: Una aproximación inicial a sus actividades en un contexto en rápida evolución; BETRISEY, Débora. Migración, comercio mayorista chino y etnicidad; CHIN et alii, op. cit.; SMITH, William. Middle Eastern Migrants in the Philippines: Entrepreneurs and Cultural Brokers; MORERAS, Jordi. ¿Ravalistán? Islam y configuración comunitaria entre los paquistaníes en Barcelona.

${ }^{15}$ Año en el que el presidente del Partido Comunista Chino, Deng Xiaoping lanzó su programa de reformas de mercado con la finalidad de crear una economía capitalista.

${ }^{16}$ ESPARGILIERE, Loup, MONNET, Theau. Los trabajadores anónimos del crecimiento chino.

${ }^{17}$ CHANG, Hsiao-hung. Fake Logos, Fake Theory, Fake Globalization.

18 DENARDI, Luciana. Casetes, redes y banquetes. Prácticas comerciales de chinos, taiwaneses y argentinos en Buenos Aires; GRIMSON, Alejandro, NG, Gustavo, DENARDI, Luciana. Las organizaciones de inmigrantes chinos en la Argentina. 
las cadenas mundiales de mercancías ${ }^{19}$. Al respecto, se ha documentado que las comunidades chinas de ultramar han encontrado en el negocio de los bazares un espacio crucial en sus procesos de incorporación económica en las sociedades de inmigración ${ }^{20}$, dando cauce a mercancías baratas, algunas de ellas falsificadas, que son atractivas en los mercados locales, especialmente para los sectores más empobrecidos de las sociedades en las que se importan estos productos.

Por otra parte, también se ha documentado que la economía del bazar organiza los modos de incorporación de otras colectividades de migrantes que desean ser parte de esta trama transnacional ${ }^{21}$. Específicamente, los migrantes africanos han encontrado en este nicho de mercado un espacio de supervivencia económica en diversos contextos a nivel internacional. Se ha documentado que migrantes originarios de Nigeria, Senegal, Mali, Gambia, Camerún y Costa de Marfil, se han reunido alrededor de los principales mercados mayoristas en los que se establecen los intermediarios principales de las mercancías que circulan por las cadenas mundiales originarias de China en localidades tan diversas como Guangzhou, Ciudad del Este, San Pablo, Hong Kong, Nueva York, Buenos Aires o Barcelona ${ }^{22}$. Si bien se ha demostrado que algunos comerciantes originarios de estos países africanos han logrado establecerse como intermediarios de las fábricas manufactureras chinas (siendo transportistas y logrando echar andar sus propios negocios) mayoritariamente sus iniciativas económicas se relacionan con la venta ambulante de dichas mercancías ${ }^{23}$. Como se describe más adelante, en la ciudad de Córdoba las iniciativas económicas de los migrantes chinos y senegaleses se integran a la globalización desde abajo a partir de su participación en el entramado comercial del bazar polirubro. Como se argumenta, se trata de relaciones comerciales desiguales, aunque complementarias, que producen posicionamientos diferenciales.

\section{La economía del bazar polirubro en la ciudad de Córdoba: relaciones entre comerciantes capitalistas y vendedores ambulantes}

La presencia de migrantes extranjeros vinculados a la economía del bazar polirubro en diversas ciudades de Argentina es un fenómeno escasamente abordado por la ciencia social nacional24. Si bien durante la década de 1990

${ }^{19}$ SMART, Alan, HSU, Jinn-You. The Chinese Diaspora, Foreing Investment and Economic Development in China.

${ }^{20} \mathrm{LI}$, Minghuan. Chinese Migration to Europe.

${ }^{21}$ BODOMO, Adams. The African Trading Communitu in Guangzhou: An Emerging Brindge for Afican-China Relations; YANG, Yang. Los comerciantes africanos en Guangzhou: rutas, razones, ganancias, sueños; STOLLER, Paul. Money, Has No Smell: The Africanization of the New York City; TULL, Denis. Chinas Engagement in Africa: Scope, Significance and Consequences.

22 Véase el trabajo de YANG, op. cit., para tener un panorama crítico de este fenómeno.

${ }^{23}$ Ibidem.

${ }^{24}$ DENARDI, op. cit; BOGADO, Laura. Migraciones internacionales. Influencia de la Migración China en el Río de la Plata; GRIMSON et alii, op. cit. 
se advirtió la presencia de migrantes de origen taiwanés y coreano en estos negocios, hoy en día éstos están regenteados casi exclusivamente por migrantes chino-cantoneses. Esta corriente migratoria cuenta con aproximadamente treinta años en el país. Datos provistos por investigaciones recientes estiman que la población originaria de China radicada en Argentina es muy superior a la que figura en el último censo poblacional del 2010 (9.000 personas nacidas en China). En cambio, se calcula que son alrededor de 120.000 personas las nacidas en China, distribuidas principalmente en la Ciudad Autónoma de Buenos Aires (CABA) y en menor medida en La Plata, Rosario, Córdoba y Tucumán ${ }^{25}$. A grandes rasgos se puede decir que esta corriente migratoria se caracteriza por tener menor capital económico que su predecesora taiwanesa de las décadas de 1980 y 1990, y menor nivel de instrucción ${ }^{26}$. Generalmente se instala el grupo familiar, incorporándose en el negocio de los supermercados.

El proceso inmigratorio de esta corriente ha estado acompañado por el establecimiento de las estratégicas relaciones comerciales entre los gobiernos de China y Argentina, especialmente desde 1996, y con mayor énfasis durante la última década. Hasta el año 2006, Argentina, tenía una relación superavitaria con China: las exportaciones argentinas hacia China se mantenían en un promedio de 6.100 millones de dólares anuales ${ }^{27}$. A partir del año 2007, la balanza se alteró y las importaciones chinas en Argentina han crecido en un promedio anual en 10.500 millones de dólares ${ }^{28}$. Actualmente, Argentina exporta a China productos no manufacturados, principalmente alimentos (soja y trigo). Por otra parte, los principales productos importados son teléfonos, computadoras, químicos, motocicletas, juguetes, manufacturas de plástico e instrumental médico ${ }^{29}$.

En Córdoba, datos oficiales muestran que para el año 2010, vivían únicamente 209 personas nacidas en China $^{30}$. Sea válido o no el dato, es claro que la población asentada en esta ciudad se ubica muy por debajo de la radicada en CABA. En tal sentido, y a diferencia de lo encontrado por las investigaciones previas, la actividad del bazar constituye la iniciativa principal de la reducida colectividad de migrantes chinos en Córdoba. En estos bazares, los comerciantes chinos venden la mercancía importada, al por menor, a los consumidores domésticos locales y al por mayor, a otros pequeños comerciantes, a grandes supermercados y a vendedores ambulantes.

\footnotetext{
${ }^{25}$ ZHANG, Tuo. Inmigrantes chinos en Argentina, una comunidad joven, dinámica y amante de una convivencia armoniosa.

${ }^{26}$ Ibidem.

${ }^{27}$ ZUAZO, Natalia, ROHMER, Matías. Un matrimonio muy desigual. Las relaciones de Argentina con el gigante asiático.

${ }^{28}$ Ibidem.

${ }^{29}$ Ibidem.

30 INDEC. Censo Nacional de Población 2010.
} 
El primer eslabón del encadenamiento en el cual se imbrican los bazares polirubro tiene su origen en las fábricas localizadas en las provincias costeras de Fujian y Guangdong. Las mismas fábricas suelen producir la totalidad de los productos manufacturados, o partes distintas de cada producto. Como señala Aguiar $^{31}$, se trata de entramados productivos que confeccionan productos baratos, en muchas ocasiones basados en la copia de otros similares. Después, diversos actores participan para consolidar las mercancías en cargamentos enviados al puerto de Buenos Aires. Por la información relevada en campo y constatada en otros lugares ${ }^{32}$ se trata de contrabandistas chinos que utilizan rutas diversas para transportar e importar la mercancía. De esta función también participan clandestinamente distintos órganos y niveles del estado-nacional argentino ${ }^{33}$. Otro eslabón crucial son los transportistas que por tierra trasladan la mercancía importada a galpones ubicados en el Área Metropolitana de Buenos Aires. Desde ahí, la mercancía es distribuida por el territorio nacional a la red de comercios chinos.

En la ciudad de Córdoba, los bazares polirubro regenteados por migrantes chinos se ubican en las avenidas principales del ejido céntrico de la ciudad. Éstos se dedican primordialmente a la venta de las mercancías del bazar. A partir de un censo que llevé a cabo en un perímetro de 120 cuadras, pude dar cuenta de la existencia de 19 bazares chinos, distribuidos a lo largo y ancho de este espacio urbano. En las entrevistas realizadas pude constatar que si bien la mayoría de estos negocios se han instalado en esta zona urbana, otros bazares se han emplazado en algunos barrios residenciales, en zonas y barrios sub-urbanos y en pequeñas localidades del interior de la provincia de Córdoba.

Todos los bazares que censé, sin excepción, se caracterizan por ser de tamaño micro y pequeño, de base familiar. En los bazares, el uso de mano de obra es escasa aunque intensiva: trabajadores familiares o nativos desempeñan labores de almacenamiento, limpieza y venta. La mayoría de las veces son regenteados por uno o dos titulares, principalmente por varones jóvenes y adultos. El propietario del bazar suele echar a andar el negocio con la ayuda de fuerza de trabajo y financiera provista por sus redes comunitarias. Una vez que el negocio se ha consolidado, el manejo del bazar pasa a manos de otro integrante de la red. De esta manera, los propietarios no suelen ser parte de los procesos de trabajo, ocupando únicamente el papel de inversores. Como otros trabajos han constatado, los propietarios financian los gastos de viaje de los

\footnotetext{
${ }^{31}$ AGUIAR, op. cit.

${ }^{32}$ Véase en la edición digital del periódico La Nación del jueves 26 de enero, los efectos políticos de una investigación en la que cayeron implicados comerciantes chinos y argentinos con mercadería no declarada: <http://www.lanacion.com.ar/1979239-el-gobierno-usaramercaderia-incautada-en-la-aduana-para-hacer-asistencia-social $>$.

33 Ibidem.
} 
recién llegados ${ }^{34}$. Asimismo, prestan o arrendan habitaciones en sus casas para la acogida de los nuevos integrantes ${ }^{35}$. Algunos de ellos emigran desde China por razones económicas. Otros cuentan con vastas experiencias migratorias y ocupacionales previas. Estos nuevos integrantes pagan con su trabajo la deuda contraída. Posteriormente, buscan acumular ahorros para echar a andar su propio negocio. Precisamente, varios de estos comerciantes que regentean los bazares polirubro fueron entrevistados para la investigación. Hasta el momento, ningún propietario ha sido entrevistado.

Es posible definir a quienes regentean estos negocios como pequeños capitalistas $^{36}$. Este concepto es útil, en tanto pone de relieve la experiencia simultánea de las personas involucradas en los negocios, como inversores y trabajadores. A diferencia de la idea de "micro-empresario", que posee una ambigua relación entre el capital-trabajo, los pequeños capitalistas chinos que manejan tiendas/bazares participan del proceso de producción e invierten en el negocio. Es decir, no sólo manejan por cuenta-propia los bazares, sino que invierten capital financiero: para incorporarse al negocio contraen deuda que deberán pagar eventualmente. Asimismo, contratan mano de obra; es decir, invierten en capital de trabajo.

Durante la investigación de campo pude constatar que los pequeños capitalistas que regentean bazares suelen participar de funciones de importación de la mercancía que venden en sus negocios. Esto es: entre diversos comerciantes contratan a un mismo agente aduanal. Esto les permite distribuirse los gastos de importación. En este punto es crucial el papel de las asociaciones de comerciantes chinos radicadas en el país ${ }^{37}$, pues éstas han funcionado como facilitadoras de los nexos entre los comerciantes y los agentes aduanales.

Pero el encadenamiento de mercancías de bazar importadas desde China no finaliza en las tiendas regenteadas por los migrantes chinos en Córdoba. El eslabón último de la cadena, y tal vez más visible que el previo, es el relacionado a la venta ambulante de dichas mercancías. Precisamente, en esta actividad se han vinculado los migrantes senegaleses, constituyéndose como revendedores de algunas las mercancías del bazar. La corriente migratoria senegalesa en Argentina es aún más reciente que la procedente de China ${ }^{38}$.

\footnotetext{
${ }^{34}$ BETRISEY, op. cit.

35 Ibidem.

${ }^{36}$ SMART, Alan, SMART, Josephine. Los pequeños capitalistas de Hong Kong.

37 GRIMSON et alii, op. cit.

${ }^{38}$ KLEIDERMACHER, Gisele. Representaciones sociales de migrantes senegaleses en Buenos Aires (1995-2014); CHEVALIER, Ellen, MORALES, Orlando. Aproximación etnográfica a la nueva migración africana en Argentina. Circulación y saberes en el caso de los senegaleses arribados en las últimas dos décadas; MAFFIA, Marta. Una contribución al estudio de la nueva inmigración africana subsahariana en la Argentina; ZUBRZYCKI, Bernarda, AGNELLI, Silvina. Allá en África, en cada barrio por lo menos hay un senegalés que sale de viaje. La migración senegalesa en Buenos Aires.
} 
Como lo han demostrado algunas investigaciones, se trata de jóvenes migrantes, predominantemente varones, miembros de la cofradía islámica mouridie, aunque también los hay pertenecientes a la cofradía tijan, así como miembros del grupo étnico diola ${ }^{39}$. Si bien se ha constatado la presencia de migrantes senegaleses en actividades relacionadas al trabajo de la construcción, carpintería y plomería, mayoritariamente se han vinculado al comercio ambulante ${ }^{40}$. En relación con la cuantía de la población senegalesa actual, no existen cifras exactas, debido a la irregularidad en el ingreso y la falta de documentación hasta fechas recientes. No obstante, y de acuerdo con datos producidos por la propia comunidad, podrían llegar a ser cerca de 5.000 los senegaleses que a la fecha se encuentran en el país, concentrándose en la CABA y en menor medida en La Plata, Mendoza, Rosario y Córdoba ${ }^{41}$.

En el centro de la ciudad de Córdoba, desde hace no más de 10 años la presencia de comerciantes ambulantes senegaleses se ha consolidado en el paisaje urbano. Los comerciantes ambulantes senegaleses, coloquialmente llamados "manteros", colocan sus puntos de venta en plazas, parques, avenidas y calles de alto tránsito y actividad comercial. En el trabajo de campo constaté que comerciantes chinos y senegaleses establecen relaciones comerciales cotidianamente. Los primeros les otorgan las mercancías en préstamo o a crédito a los segundos, que deberán pagar semanal o mensualmente. Las mercancías de interés comercial son bolsos, carteras, bijouterie, relojes, anteojos y pañuelos. Estos convenios son informales y varían dependiendo de los acuerdos establecidos entre las partes.

Por otra parte, he podido distinguir dos modalidades de venta ambulante. En primer lugar, existen puntos de venta callejera "fijos". En estos puntos de venta se emplazan los migrantes pioneros que "ganaron" esos espacios estratégicos en algunas calles, avenidas y plazoletas. Los migrantes pioneros, con mayor experiencia, suelen ser los "jefes". Organizan las relaciones comerciales de otros integrantes de la comunidad, que no hablan español con fluidez. Por ejemplo, entregan mercadería a préstamo a los recién llegados, que será devuelta al tiempo. Son también quienes deciden los tiempos que estarán en uno u otro punto de venta, y son quienes llevan a cabo las transacciones con las fuerzas del orden que controlan el espacio público. Si bien algunos de estos "jefes" establecen relaciones comerciales con los bazaristas chinos, la mayoría de estos comerciantes poseen nexos con otros eslabones de la cadena que les venden directamente la mercancía importada. Estos eslabones se ubican, principalmente en Buenos Aires y en diversos pasos aduanales en la Provincia de Mendoza.

\footnotetext{
39 Ibidem.

40 KLEIDERMACHER, op. cit.

${ }^{41}$ Ibidem, p. 67.
} 
En segundo lugar, existen puntos de venta "móviles". Éstos, están personificados por migrantes con menor experiencia. Circulan por diversas zonas comerciales de la ciudad. Estos circuitos no son aleatorios, sino que están determinados diariamente por los vendedores ambulantes. Al igual que aquellos incorporados a los puntos de puesta fijos, estos comerciantes se agrupan en grupos de dos o más. Así se protegen mutuamente de los controles policiales y de posibles robos. En base a la información relevada, la mayoría de los comerciantes ambulantes senegaleses radicados en Córdoba se han incorporado a esta modalidad de venta. En consecuencia, los comerciantes chinos se constituyen como sus principales intermediarios.

La configuración de estas relaciones comerciales permite enunciar que estamos ante la presencia de un sistema de relaciones comerciales complementarias, aunque desiguales. Son complementarias pues para ambas actividades su principal mercado de consumo se encuentra en los sectores populares. Adicionalmente, entre estas actividades se establecen relaciones comerciales crediticias: los bazaristas otorgan las mercancías tanto a préstamo o bajo consignación en periodos semanales o mensuales. Así, mientras los vendedores ambulantes deben pagar una comisión por cada venta o proporción de ventas, los comerciantes chinos devienen en sus acreedores permanentes.

Por otro lado, estas relaciones reproducen experiencias económicas desiguales: las primeras asociadas a las estrategias capitalistas, las segundas a las de supervivencia. Como se ha podido observar, la participación de los comerciantes de origen chino se presenta primordialmente en las tiendas/ bazares. Al participar tanto del proceso de trabajo como de la inversión en estos negocios, es posible concebirlos como micro y pequeños capitalistas quienes no se limitan a las funciones propias de las tiendas, sino que algunos participan directamente de la importación, eliminando costos de intermediación. En cambio, la organización de los comerciantes ambulantes senegaleses está estratificada por partida doble, especialmente para aquellos comerciantes menos experimentados que al no poseer nexos con otros eslabones del encadenamiento, se subordinan a los comerciantes senegaleses con más experiencia y a los bazares regenteados por los pequeños capitalistas chinos. Esto los posiciona en la base del entramado comercial mundial del bazar, condicionando sus circunstancias económicas y de vida cotidiana.

\section{Conclusiones}

Mediante la instrumentación de un enfoque especialmente atento a los procesos económicos relacionados a la globalización desde abajo, en este artículo he analizado la estructuración de dos actividades personificadas por migrantes de origen chino y senegalés, vinculadas al entramado comercial de los bazares polirubro. A través del desarrollo de un abordaje etnográfico, he 
descrito dos procesos económicos experimentados por estos sujetos, ubicados en posiciones subalternas de la economía global no hegemónica. Haciendo uso de una perspectiva centrada en las cadenas mundiales de mercancías por las que circulan los bienes que estos sujetos comercializan, he podido dar cuenta que, por un lado, los bazares/tiendas polirubro regenteados por comerciantes de origen chino y, por otro, las actividades de venta ambulante Ilevadas a cabo por comerciantes senegaleses, constituyen dos eslabones de un entramado comercial que tiene su origen en las provincias manufactureras del sureste chino y su fin en las prácticas de consumo en las calles de la ciudad de Córdoba, Argentina.

Sin embargo, el aporte principal del trabajo es que mediante de un descripción de la configuración de estas actividades, se ha encontrado que ambas son al mismo tiempo complementarias, aunque profundamente desiguales entre sí: aunque las dos actividades forman parte de un mismo encadenamiento de mercancías, los bazares constituyen iniciativas económicas en las que se invierten capitales financieros y de trabajo. Quienes participan de este eslabón no se limitan a la venta de las mercancías sino que se involucran en otras actividades del encadenamiento. Adicionalmente, se ha encontrado que la mayoría de los eslabones que configuran al encadenamiento están personificados por personas, empresas y organizaciones chinas, localizadas tanto en su territorio nacional como en las comunidades de ultramar, lo que ha dado forma a un entramado comercial de base comunitaria. En segundo lugar, se ha podido ver que buena parte de los vendedores ambulantes senegaleses, especialmente los recién llegados a la ciudad, al no disponer de capitales semejantes, dependen comercialmente de los bazares regenteados por comerciantes chinos para surtirse de las mercancías que comercializarán en las calles de la ciudad. Es posible enunciar que ello esté contribuyendo en la configuración de experiencias económicas subordinadas a los bazares chinos, y al mismo tiempo estrechamente dependientes de los comerciantes senegaleses con mayor experiencia y acceso a la importación directa de las mercancías de bazar.

Otro aporte de este trabajo es que ha desplegado una mirada teóricometodológica des-etnizada, a partir de la cual el objeto de estudio no han sido los sujetos, sino las relaciones sociales que establecen los sujetos migrantes durante sus procesos de incorporación. Esta mirada ha permitido ampliar el rango analítico de la investigación, pues al no tomar en cuenta como variable de recorte del objeto de estudio a las características étnico-nacionales de los sujetos, el ojo se ha puesto en la diversidad que toman los procesos de incorporación económica. Ello ha dado pie a la consideración simultánea de las dos prácticas económicas bajo estudio. Finalmente, la particularidad de esta investigación es que ésta ha sido desarrollada en un entorno societal en el que previamente no se habían atendido los procesos económicos experimentados por migrantes 
extranjeros que no forman parte de las corrientes migratorias cuantitativamente principales. Esto nos convoca a mirar en ulteriores oportunidades los efectos que estas condiciones de producción están teniendo sobre las trayectorias de vida, así como sobre las construcciones subjetivas de aquellos que llevan a cabo estas prácticas económicas en la ciudad de Córdoba.

\section{Bibliografía}

AGUIAR, José Carlos. "Vienen de China". Los CD piratas en México desde una perspectiva transnacional. In ALBA VEGA, Carlos; RIBEIRO, Gustavo; MATHEWS, Gordon (coords.). La globalización desde abajo. La otra economía mundial. México: Fondo de Cultura Económica, 2015, p. 81-109.

ALBA VEGA, Carlos; RIBEIRO, Gustavo; MATHEWS, Gordon (coords.). La globalización desde abajo. La otra economía mundial. México: Fondo de Cultura Económica, 2015.

APPADURAI, Arjun. Grassroots Globalization and the Research Imagination. Public Culture, v. 12 n. 1, 2000, p. 1-19.

BETRISEY, Débora. Migración, comercio mayorista chino y etnicidad. CIDOB d'Afers Internacionals, n. 78, 2007, p. 77-95.

BODOMO, Adams. The African Trading Communitu in Guangzhou: An Emerging Brindge for Afican-China Relations. China Quarterly, v. 203, 2010, p. 693-707.

BOGADO, Laura. Migraciones internacionales. Influencia de la Migración China en el Río de la Plata. Tesis de maestría en Relaciones Internacionales. La Plata: UNLP, 2012.

CHANG, Hsiao-hung. Fake Logos, Fake Theory, Fake Globalization. Inter-Asia Cultural Studies, v. 5, n. 2, 2004, p. 222-236.

CHEVALIER, Ellen; MORALES, Orlando. Aproximación etnográfica a la nueva migración africana en Argentina. Circulación y saberes en el caso de los senegaleses arribados en las últimas dos décadas. Astrolabio, n. 8, 2012, p. 381-405.

CHIN, Ku-Sup; YOON, In-Jin; SMITH David. Immigrant Small Business and International Economic Linkage. A Case of the Korean Wig Business in Los Angeles, 1968-1977. The International Migration Review, v. 30, n. 2, 1996, p. 485-510.

DENARDI, Luciana. Casetes, redes y banquetes. Prácticas comerciales de chinos, taiwaneses y argentinos en Buenos Aires. Etnografías Contemporáneas, v. 2, n. 1, 2016, p. 134-160.

ESPARGILIERE, Loup; MONNET, Theau. Los trabajadores anónimos del crecimiento chino. Le monde Diplomatique, Edición 209 (noviembre), 2016.

GAGO, Verónica. La Razón Neoliberal. Economías barrocas y pragmática popular. Buenos Aires: Tinta Limón Ediciones, 2014.

GLICK, Nina; CAGLAR, Ayse; GULDBRANDSEN, Thaddeus. Beyond the ethnic lens: Locality, globality, and born-again incorporation. American Ethnologist, v. 33, 2006, p. 612-633. 
GRIMSON, Alejandro; NG, Gustavo; DENARDI, Luciana. Las organizaciones de inmigrantes chinos en la Argentina. Migración y Desarrollo, v. 14, n. 16, 2016, p. 25-74.

INDEC. Censo Nacional de Población 2010. Disponible en: < http://www.indec. gob.ar/>.

KLEIDERMACHER, Gisele. Representaciones sociales de migrantes senegaleses en Buenos Aires (1995-2014). Revista Mexicana de Sociología, v. 79, n. 1, 2017, p. 65-92.

LI, Minghuan. Chinese Migration to Europe: An Overview. The China monitor, n. 53, 2010, p. 4-8.

LÓPEZ SALA, Ana; SÁNCHEZ, Valeriano. Los comerciantes sindhis en Canarias. Relaciones transnacionales y actividad económica. CIDOB d'Afers Internacionals, n. 92, 2010, p. 139-159.

MAFFIA, Marta. Una contribución al estudio de la nueva inmigración africana subsahariana en la Argentina. Cuadernos de Antropología Social, n. 31, 2010, p. 7-32.

MEZZADRA, Sandro. Capitalismo, migraciones y luchas sociales. La mirada de la autonomía. Nueva Sociedad, n. 237, 2012, p. 159-178.

MORERAS, Jordi, ¿Ravalistán? Islam y configuración comunitaria entre los paquistaníes en Barcelona. CIDOB d'Afers Internacionals, n. 68, 2005, p. 119-132.

RIBEIRO, Gustavo. Non hegemonic globalizations. Alternativa transnational Processes and agents. Anthropological Theory, v. 9 n. 3, 2009, p. 1-33.

RIBEIRO, Gustavo. Economic Globalization from Below. Etnográfica, v. 10, n. 2, 2006, p. 233-248.

SMART, Alan; HSU, Jinn-You. The Chinese Diaspora, Foreing Investment and Economic Development in China. The Review of International Affairs, v. 3 n. 4, 2004, p. 544-566.

SMART, Alan; SMART, Josephine. Los pequeños capitalistas de Hong Kong. In ALBA VEGA, Carlos; RIBEIRO, Gustavo; MATHEWS, Gordon (coords.). La globalización desde abajo. La otra economía mundial. México: Fondo de Cultura Económica, 2015, p. 191-221.

SMITH, William. Middle Eastern Migrants in the Philippines: Entrepreneurs and Cultural Brokers. Asian Journal of Social Science, v. 32, n. 3, 2004, p. 425-457.

SOlÉ, Carlota; PARELLA, Sonia; CALVACANTI, Leonardo. El empresariado inmigrante en España. Colección de Estudios Sociales, n. 21, Barcelona: Fundación La Caixa, 2007.

STOLLER, Paul. Money, Has No Smell: The Africanization of the New York City. Cambridge: The Chicago Press University, 2002.

TSENG, Yen. Beyond "Little Taipei": The Development of Taiwanese Immigrant Businesses in Los Angeles. The International Migration Review, v. 29, n. 1, 1995, p. 33-58.

TULL, Denis. Chinas Engagement in Africa: Scope, Significance and Consequences. Modern African Studies, v. 44, n. 3, 2006, p. 459-479. 
VUDDAMALAY, Vasoodeven. Empresarios indios en Francia: Una aproximación inicial a sus actividades en un contexto en rápida evolución. CIDOB d'Afers Internacionals, n. 92, 2010, p. 99-118.

YANG, Yang. Los comerciantes africanos en Guangzhou: rutas, razones, ganancias, sueños. In ALBA VEGA, Carlos; RIBEIRO, Gustavo; MATHEWS, Gordon (coords.). La globalización desde abajo. La otra economía mundial. México: Fondo de Cultura Económica, 2015, p. 284-313.

YOON, In-Yin. The Changing Significance of Ethnic and Class Resources in Immigrant Businesses. The Case of Korean Immigrant Businesses in Chicago. The International Migration Review, v. 25, n. 2, 1991, p. 303-332.

ZHANG, Tuo. Inmigrantes chinos en Argentina, una comunidad joven, dinámica y amante de una convivencia armoniosa. Revista de la COPPPAL, 2007, s/n.

ZUAZO, Natalia; ROHMER, Matías. Un matrimonio muy desigual. Las relaciones de Argentina con el gigante asiático. Explorador China, n. 1, 2013, p. 65-67.

ZUBRZYCKI, Bernarda; AGNELLI, Silvina. Allá en África, en cada barrio por lo menos hay un senegalés que sale de viaje. La migración senegalesa en Buenos Aires. Cuadernos de Antropología Social, n. 29, 2009, p. 135-152.

Recibido para publicación en 31.01.2017

Aceptado para publicación en 10.03.2017

Received for publication in January 315t, 2017

Accepted for publication in March 10 ${ }^{\text {th }}, 2017$

ISSN impresso 1980-8585

ISSN eletrônico 2237-9843

http://dx.doi.org/10.1590/1980-85852503880004912 